\title{
Separation Efficiency of Livestock Manure Biogas by Membrane
}

\author{
Jong Eon Kim, Sang Yun Lee, Chung Seop Lee and Hyeon Tae Kim* \\ Department of Bio-Industrial Machinery Engineering, Gyeongsang National University \\ (Institute of Agriculture \& Life Science) \\ Jinju 660-701, Korea
}

\begin{abstract}
In this study, biogas generated from the anaerobic digestion of animal manure was purified and separated and the hollow fiber membrane was prepared by the phase separation of cellulose triacetate (CTA) method to separate the biogas. The product was used in the experiment because the separation of gas by using a gas permeation rate of the hollow fiber membrane was produced. A hollow fiber gas separation membrane module using the hollow fiber membrane in the test was produced and biogas generated in the anaerobic digester was purified using a gas separation membrane module. The separation and purification of the resulting biogas was generated from the livestock manure. The isolation and purification of the resulting biogas was $\mathrm{CH}_{4} 94.2 \%, \mathrm{CO}_{2} 92.0 \%$ and recovery efficiency was $\mathrm{CH}_{4} 102.1 \%, \mathrm{CO}_{2} 83.5 \%$. Siloxane $0.1 \mathrm{PPM}$ or less, $\mathrm{H}_{2} \mathrm{~S} 3 \mathrm{PPM}$ or less were fed, Dewpoint $-20^{\circ} \mathrm{C}$ below by controlling the biogas supplied to the gas separation membrane module at this time and the operating pressure of the gas separation membrane module was 4 7Bar, in the range of temperature is $15 \sim 45^{\circ} \mathrm{C}$. Therefore, it was separated in a high concentration of $\mathrm{CO}_{2} 92.0 \% \mathrm{CH}_{4} 94.2 \%$, compared to the composition of the biogas $\left(\mathrm{CH}_{4} 68 \%, \mathrm{CO}_{2} 28 \%, \mathrm{O}_{2} 0.6 \%, \mathrm{H}_{2} \mathrm{~S} 1 \mathrm{PPM}\right.$ or less, Balance $\left.2.7 \%\right)$ before carrying out the separation and purification test of biogas produced in the study using the gas separation membrane module. In addition, the $\mathrm{CH}_{4}$ and $\mathrm{CO}_{2}$ transfer efficiency was maximized and saved in the stainless steel tank.
\end{abstract}

Keywords : Cellulose triacetate, $\mathrm{CO}_{2}, \mathrm{CH}_{4}$, separation, purification, transfer efficiency

\section{INTRODUCTION}

Currently, the total global oil reserve has reached an alarming level (1trillion 4,747 billion barrels), which would serve the world only for the next 37.5 years (Petroleum Society, 2000).According to BP statistics 93 edition, Korea was ranked 10 after Canada by 164 million barrels of oil per day. The energy consumption of Korea was high and was ranked 11 in 1994 (Oil Association, 1994). The energy consumption of South Korea is about twice as high as that of the developed countries and the industry sector of the country depends largely on oil resources for their energy supply, which also has a great impact on the greenhouse gas emissions.

With this high energy requirement, Korea suffers from the dearth of fossil fuel energy resources (anthracite coal is the only fossil fuel found in Korea). Therefore, 97\% of Korea's energy requirement, including its agricultural sector, is met by foreign sources. It is important that Korea must develop a strategy for dealing with international environmental issues effectively.

\footnotetext{
* Corresponding Author: bioani@gnu.ac.kr
} 
Dumping of wastes in ocean has been banned in 2013 due to negative environmental effects. Along with that, recycling of food wastes and livestock manure has been highly encouraged as an alternative source of energy (Hauk circle, 2012). The current study aims to separate methane and carbon dioxide from the biogas, which was produced from the anaerobic digestion of animal manure.

\section{Experimental setup}

\section{METHODOLOGY}

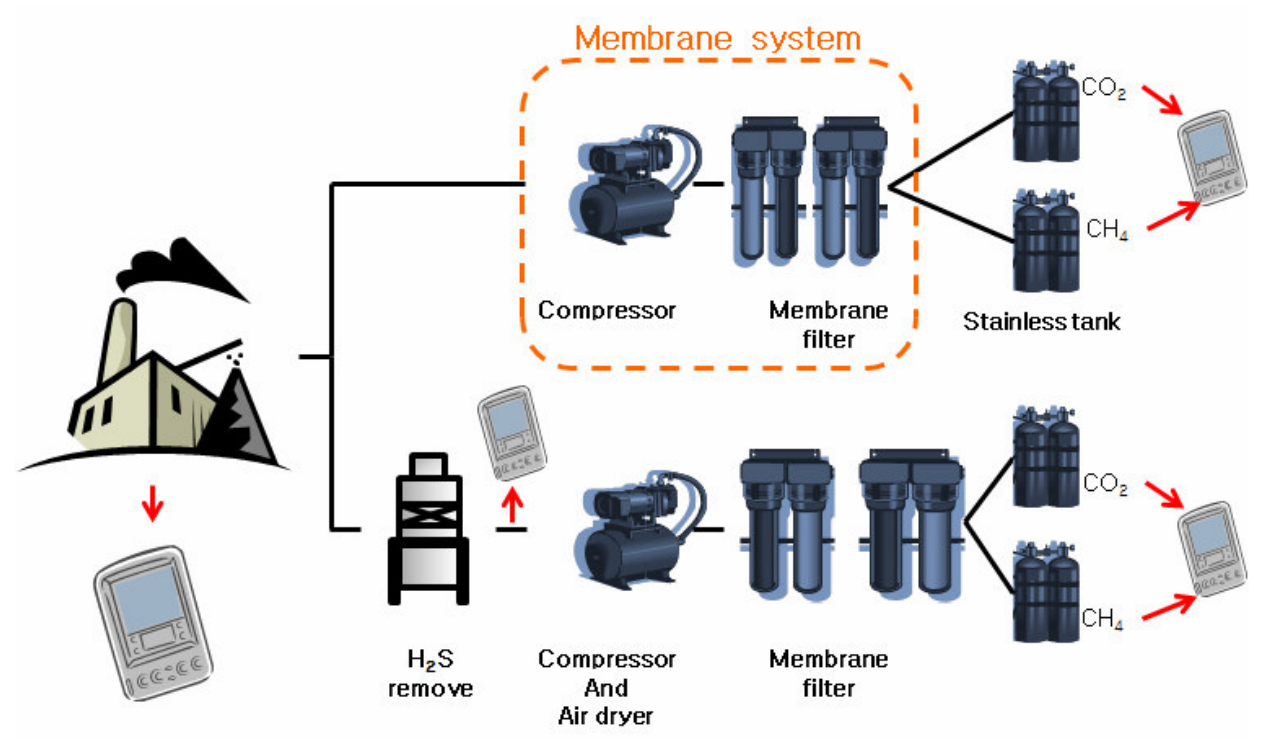

Fig. 1. Bio-gas separation and purification process flow chart.

The plants from where biogas was obtained in the present work are located in (U)proenvironment Daehyeon Green, Sintaein-eup, Jeongeup-si, Jeollabuk-do, Korea. Table 1 shows the gas composition used for this study.

Table 1. Component of biogas collected in a biogas plant

\begin{tabular}{||l|c|c|c|c|c||}
\hline & $\mathrm{CH}_{\mathbf{4}}(\boldsymbol{\%})$ & $\mathrm{CO}_{\mathbf{2}}(\boldsymbol{\%})$ & $\mathrm{O}_{\mathbf{2}}(\boldsymbol{\%})$ & $\mathbf{H}_{\mathbf{2}} \mathrm{S}(\mathrm{PPM})$ & Balance(\%) \\
\hline Component & 68 & 28 & 0.6 & 1 & 2.7 \\
\hline \hline
\end{tabular}

To remove hydrogen sulfide and moisture from the biogas, the biogas was passed through the desulfurization unit and the air dryer, respectively so that the dew point of the dried biogas was reduced to $-20{ }^{\circ} \mathrm{C}$. The components removed from the biogas were measured with a biogas simple meter. An optimum operating pressure of 4-7 bar was maintained at the gas separation membrane by an air compressor to continue the separation and purification procedure. 
The gas separating membrane module was placed in stainless steel reservoir tanks to prevent any reaction by the purified $\mathrm{CH}_{4}$ and $\mathrm{CO}_{2}$. The stored $\mathrm{CH}_{4}$ and $\mathrm{CO}_{2}$ gases were finally measured by biogas simple meter.

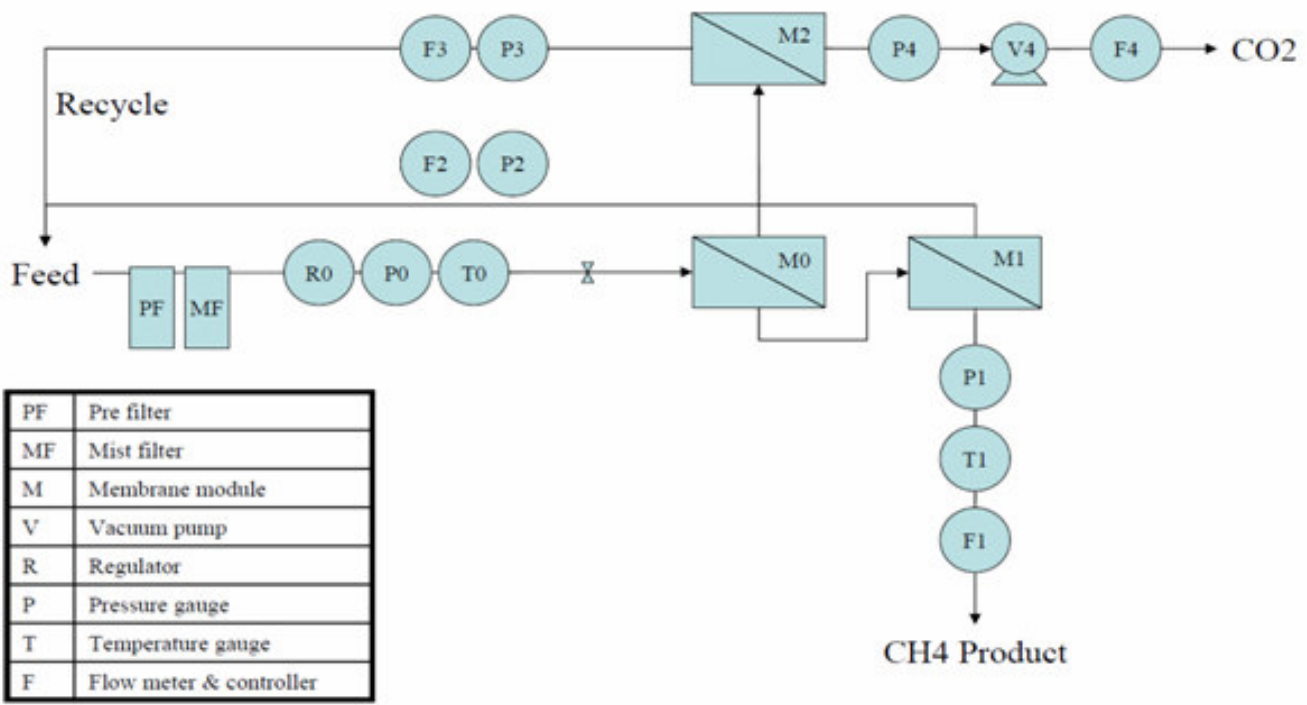

Fig. 2. Gas flow inside the membrane module.

The flowchart, as shown in Figure 2 and the internal gas separation membrane module were designed for biogas generated from livestock manure and the tablets.

The system is composed of three gas separators and the gas separation membranes are connected to each other. The separation and purification relied on the principle that methane permeates at a slower rate than $\mathrm{CO}_{2}$ through the gas separation membrane. In the first module and the third module to the module $\mathrm{CH}_{4}$ is transmitted faster than the $\mathrm{CO}_{2}$ permeable, $\mathrm{M} 0 \mathrm{CO}_{2}$ is then transmitted from the small amount of the remaining is supplied to the M1 $\mathrm{CO} 2$ and $\mathrm{CH}_{4}$ again. The separating gas in the same way and $\mathrm{MO}$ is generated $\mathrm{CH}_{4}$ in the high-concentration gas.

On the other hand, we generates a high-concentration $\mathrm{CO} 2$ gas permeation rate of the hollow fibre membrane by the difference between the gas in the M2 transmitted and M0 CO2 gas. 


\section{Experimental devices}

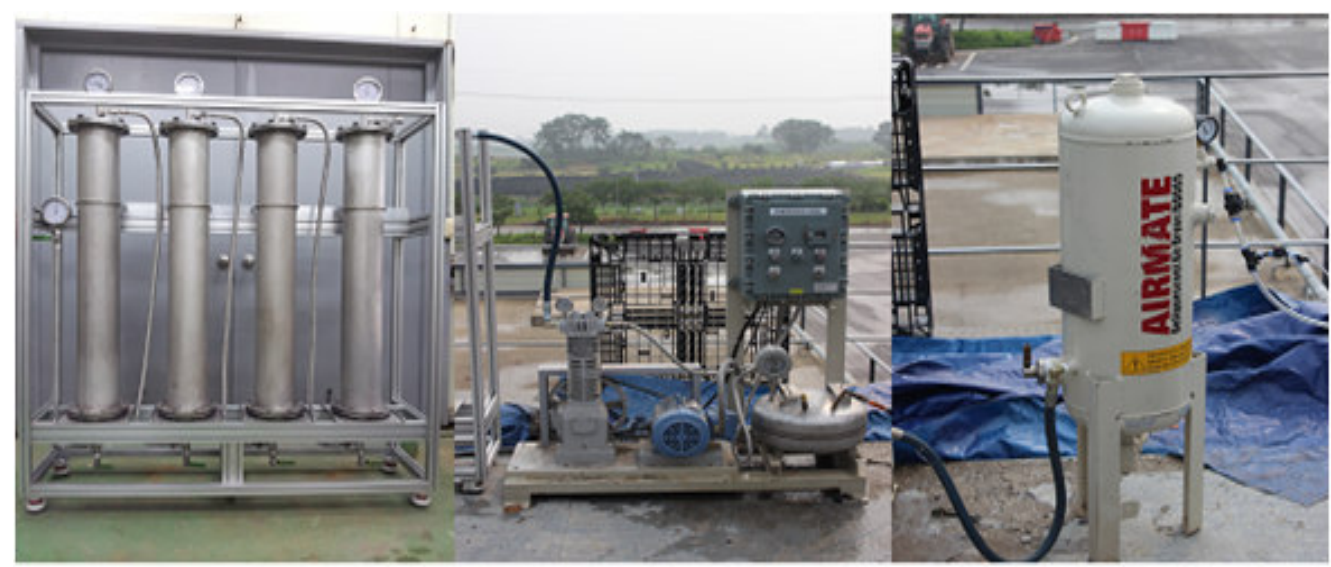

Fig. 3.

Desulfurizer

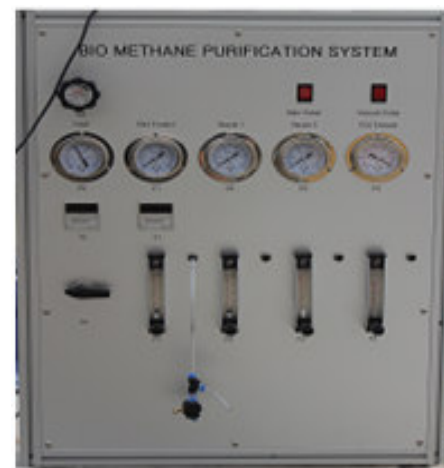

Fig. 6.

Gas separation

membrane module
Fig. 4.

Air compressor

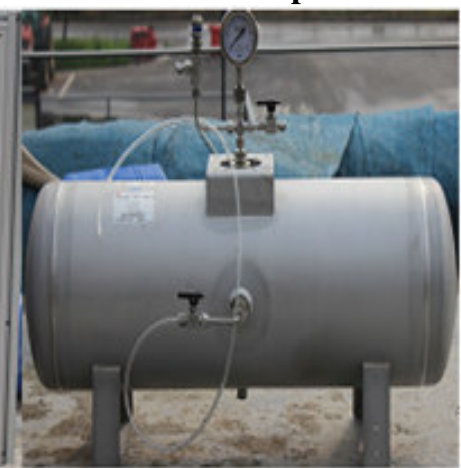

Fig. 7.

Stainless steel tank
Fig. 5.

Air dryer

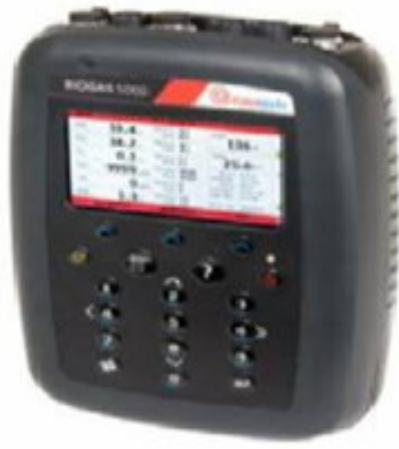

Fig. 8.

Simple meter of biogas

Fig. 3-8 shows all the different devices used in the current work for biogas treatment.

Less than $0.1 \mathrm{PPM}_{2} \mathrm{~S}$ and $3 \mathrm{PPM}$ siloxane were maintained using a desulfurizer (ESC150A-01, ESC Ltd., Republic of Korea,) and an air compressor (TFPS55-10, Ernest Iwata Korea Ltd., Republic of Korea). The air dryer (*BD005, Baumann Ltd., Republic of Korea) with a pressure of 10 bar was also used in this study. The gas membrane separation module (MC-1512A, Air Lane Ltd., Republic of Korea) was used to separate and purify the biogas, which was then dried in the air-dryer and stored in stainless steel tanks (STS304, Dongying Entec, Republic of Korea Mat'l). The bio-gas meter (Biogas 5000, Country Science, Republic of Korea) was used to measure the remaining components in the biogas, e.g. $\mathrm{O}_{2}$, $\mathrm{CH}_{4}, \mathrm{CO}_{2}$, and $\mathrm{H}_{2} \mathrm{~S}$.

\section{Experimental equation}

In this study, the amount of the biogas supplied to the gas separation membrane module was obtained from Eq. 1. The recovery efficiency of the $\mathrm{CH}_{4}$ and $\mathrm{CO}_{2}$ was calculated using Eq. 2 
$\& 3$, respectively. The recovery efficiency denotes the amount of gas recovered after being separated by the gas separation membrane module.

$$
\text { Feed }=\text { Permeate }+ \text { Retentate }
$$

Where, Feed $=$ Supplied gas to the hollow fibre membrane module

Permeate $=$ Amount of permeate gas

Rententate $=$ Amount of rententate gas

$$
\mathrm{CH}_{4} \text { recoveryefficiency }(\%)=\frac{P \times C P_{\mathrm{CH}_{4}}}{F \times C F_{\mathrm{CH}_{4}}} \times 100
$$

Where, $\mathrm{CH}_{4}$ recovery efficiency = Recovery efficiency of $\mathrm{CH}_{4}$ gas

$\mathrm{P}=$ Amount of permeate gas

$\mathrm{CP}_{\mathrm{CH} 4}=$ Concentration of methane before separation

$\mathrm{F}=$ Supplied gas to the hollow fibre membrane module

$\mathrm{CF}_{\mathrm{CH} 4}=$ Concentration of methane after separation

$$
\mathrm{CO}_{2} \text { recoveryefficiency }(\%)=\frac{\mathrm{P} \times \mathrm{CP}_{\mathrm{CO}_{2}}}{\mathrm{~F} \times \mathrm{CF}_{\mathrm{CO}_{2}}} \times 100
$$

Where, $\mathrm{CO}_{2}$ recovery efficiency $=$ Recovery efficiency of $\mathrm{CO}_{2}$ gas

$\mathrm{P}=$ Amount of permeate gas

$\mathrm{CP}_{\mathrm{CO} 2}=$ Concentration of carbon dioxide before separation

$\mathrm{F}=$ Supplied gas to the hollow fibre membrane module

$\mathrm{CF}_{\mathrm{CO} 2}=$ Concentration of carbon dioxide after separation

\section{RESULTS AND DISCUSSION}

The results of this experiment are shown in Figure 9. The bio-gas used in the experiment was composed $\mathrm{CH}_{4}(68 \%), \mathrm{CO}_{2}(28 \%), \mathrm{H}_{2} \mathrm{~S}(1 \mathrm{PPM}), \mathrm{O}_{2}(0.6 \%)$, Balance (2.7\%) prior to passing through the gas separation membrane module.

Methane, the biogas was passed through the gas separation membrane and the composition of the methane fraction was $\mathrm{CH}_{4} 94.2 \%, \mathrm{CO}_{2} 0.2 \%, \mathrm{O}_{2} 0 \%, \mathrm{H}_{2} \mathrm{~S} 0$ PPM, Balance $4.8 \%$. Similarly, after removing the $\mathrm{CO}_{2}$ fraction, the composition of the fraction was $5.8 \%, \mathrm{CO}_{2}$ $92.0 \%, \mathrm{O}_{2} 0.6 \%, \mathrm{H}_{2} \mathrm{~S} 2 \mathrm{PPM}$, Balance $1.6 \%$. Siloxane also controlled to 0.1 PPM or less, the Dewpoint below $-20{ }^{\circ} \mathrm{C}, \mathrm{H}_{2} \mathrm{~S} 3 \mathrm{PPM}$ or less, the operating pressure of the device at 4-7 Bar and temperature within $15-45^{\circ} \mathrm{C}$ at this time. The amount of gas supplied to the gas separation membrane module can be obtained from Eq. 1. The residual amount and the permeated amount of gas can be obtained from Eq. 4 . 


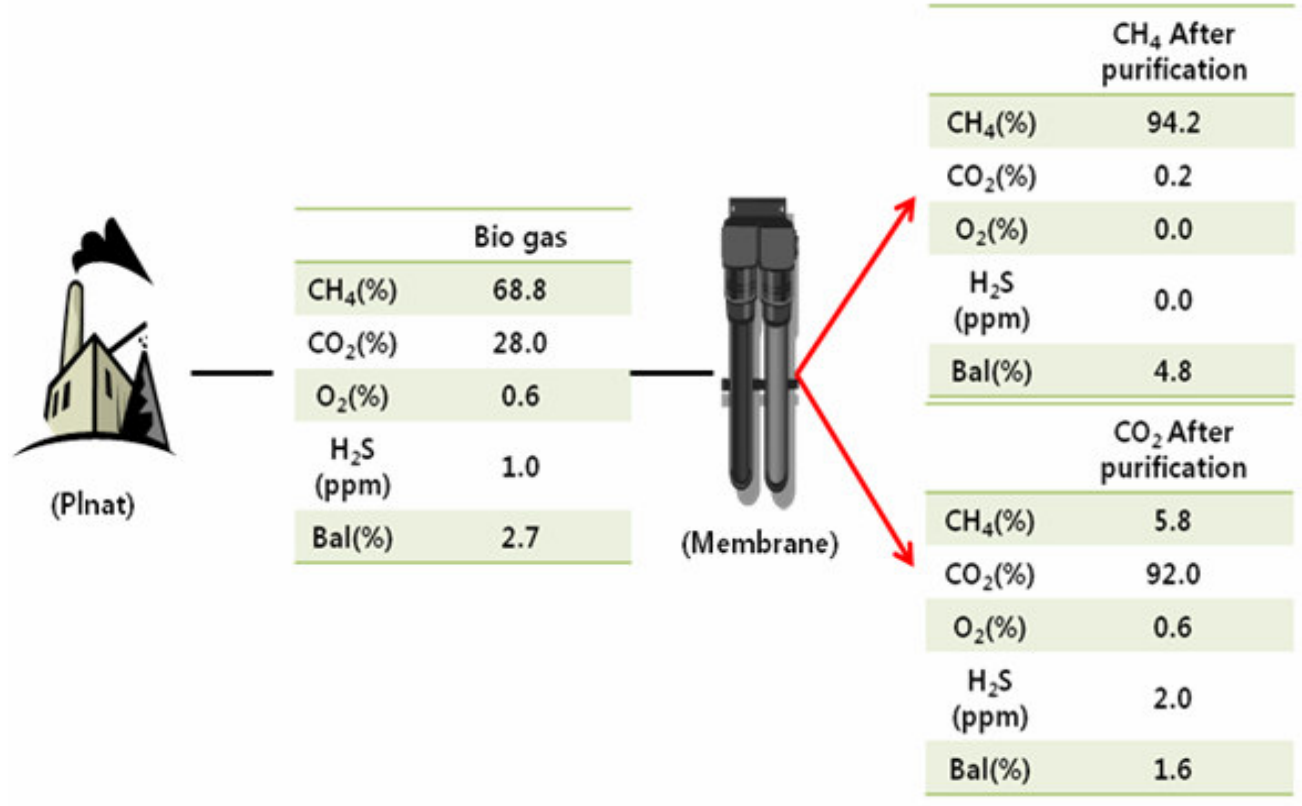

Fig. 9. Separation of bio-gas by a gas separation membrane module and refining results

$$
\begin{aligned}
\text { Feed } & =\text { permeate }+ \text { Exaust } \\
& =22 L+7.5 L=29.5 L
\end{aligned}
$$

Eq. ... 4

The recovery efficiency of the gas separated by the gas separation membrane module was calculated from Eq. 2. Calculation of the recovery efficiency of $\mathrm{CH}_{4}$ gas is shown in Eq. 5 .

$$
\begin{aligned}
& \mathrm{CH}_{4} \text { recoveryefficiency }(\%)=\frac{P \times C P_{C_{4}}}{F \times C F_{C H_{4}}} \times 100 \\
& \therefore C H_{4} r e=\frac{(22 l \times 94.2 \%)}{(29.5 l \times 68.8 \%)} \times 100=102.1 \%
\end{aligned}
$$

Similarly, the recovery efficiency of $\mathrm{CO}_{2}$ was calculated from Eq. 3 and the calculation has been shown in Eq. 6.

$$
\begin{aligned}
& \mathrm{CO}_{2} \text { recoveryefficiency }(\%)=\frac{P \times C P_{\mathrm{CO}_{2}}}{F \times C F_{\mathrm{CO}_{2}}} \times 100 \\
& \therefore \mathrm{CO}_{2} \text { re }=\frac{(7.5 l \times 92 \%)}{(29.5 l \times 28 \%)} \times 100=83.5 \%
\end{aligned}
$$

Biogas was fed at the rate of $29.5 \ell$ obtained in addition the amount of gas generated in the transmission portion and the remaining portion of the gas separation membrane module, the residue from the gas separation membrane module $22 \ell$ and $7.5 \ell$ has been separated is transmitted. Out of $29.5 \mathrm{~L}$ gas fed, $22.5 \mathrm{~L}$ has been successfully separated into methane and 
carbon dioxide, and the rest 7.5 L was unaffected. A high recovery efficiency of $102.1 \%$ of the biogas was achieved. To address the anomalous value (more than 100\%), the value was corrected for recovery efficiency of $\mathrm{CH}_{4}$. It appeared that some errors are included in $\mathrm{N}_{2}$ and $\mathrm{CH}_{4}$ recovery data. On the other hand, $\mathrm{CO}_{2}$ showed a recovery efficiency of $83.5 \%$.

\section{CONCLUSIONS}

The results obtained in this work revealed that an efficient separation of methane and carbon dioxide was achieved from the biogas- $\mathrm{CH}_{4} 94.2 \%, \mathrm{CO}_{2} 92.0 \%$. High concentration $\mathrm{CH}_{4}$ $102.1 \%, \mathrm{CO}_{2} 83.5 \%$ isolated and purified in the recovery efficiency. This would have been included $\mathrm{N}_{2}$ and $\mathrm{CH}_{4}$ aqueous phase trace. The future plan is to work on optimization of separation conditions of biogas through gas separation membrane module.

\section{ACKNOWLEDGEMENTS}

In this study was performed support of Rural Development Administration Research Project (PJ010000).

\section{REFERENCES}

Hyeongcheol Go. (2011). Separation and Purification of Bio Gas by Hollow Fibre Gas Separation Membrane Module. 5.

Korea Petroleum Association. (1994). Oil consumption is the country to some extent?, 1-2.

Korea Petroleum Association. (2000). Undiscovered oil reserves in the world

Taesu Song. (2000). Preferred direction of environmental policy and international environmental issues in Korea. 2, 1 - 20.

Ukwon Ha. (2012). Since long-term ocean dumping ban cattle manure recycling measures.

Yunji Min. (2014). An energy efficiency analysis of Korea based on the energy dependence and independence rate. 2, $1-29$. 